\title{
The Use of Hormonal Contraceptives and Preeclampsia among Ghanaian Pregnant Women
}

\section{Listowell Asare ${ }^{1}$, George A. Asare ${ }^{2}$, William K. B. A. Owiredu' ${ }^{1}$ Christian Obikorang1, Efua Appiah², Worlanyo Tashie ${ }^{2}$, Leila Seidu ${ }^{3}$}

\author{
${ }^{1}$ Department of Molecular Medicine, School of Medical Sciences, Kwame Nkrumah University \\ of Science and Technology, Kumasi, Ghana \\ ${ }^{2}$ University of Ghana School of Biomedical and Allied Health Sciences (SBAHS), Accra, Ghana \\ ${ }^{3}$ Comboni Hospital, Sogakope, Ghana \\ Email: *gasare@chs.edu.gh
}

How to cite this paper: Asare, L., Asare, G.A., Owiredu, W.K.B.A., Obikorang, C., Appiah, E., Tashie, W. and Seidu, L. (2021) The Use of Hormonal Contraceptives and Preeclampsia among Ghanaian Pregnant Women. Open Journal of Obstetrics and Gynecology, 11, 419-433.

https://doi.org/10.4236/ojog.2021.114041

Received: March 13, 2021

Accepted: April 22, 2021

Published: April 25, 2021

Copyright $\odot 2021$ by author(s) and Scientific Research Publishing Inc. This work is licensed under the Creative Commons Attribution International License (CC BY 4.0).

http://creativecommons.org/licenses/by/4.0/

\begin{abstract}
Hormonal contraceptives ( $\mathrm{HC}$ ) are thought to play a role in the pathogenesis of cardiovascular diseases. The study evaluated the use of $\mathrm{HC}$ as a primary cause of preeclampsia (PE) among Ghanaians. This study comprised 30 preeclamptic women and 30 healthy normotensive pregnant women with over 20 weeks of gestation at the Comboni Hospital, Ghana using a randomized case-control study. Blood pressure, weight, height, socio-demographics, medical and previous obstetric history were taken and recorded. Blood samples were collected for the estimation of homocysteine and fasting lipids. Estimated foetal weight (EFW) and infant birthweight (BWT) were obtained from maternal records. This study was carried out in 2019. 80.0\% of women with PE used the hormonal contraceptive "depot medroxyprogesterone acetate" (DMPA) prior to pregnancy. The use of DMPA was associated with about thirty-fold increase in the odds of developing PE (OR $=29.71, \mathrm{p}<$ $0.001)$. Systolic blood pressure $(\mathrm{P}<0.001)$, diastolic blood pressure $(\mathrm{P}<$ $0.001)$, triglycerides $(\mathrm{P}=0.024)$, LDL-C $(\mathrm{P}=0.026)$, and homocysteine levels $(\mathrm{P}<0.001)$ were significantly elevated in the $\mathrm{PE}$ cases than the normal pregnant (NP) women, whilst EFW $(\mathrm{P}<0.001)$, BWT $(\mathrm{P}<0.001)$ and HDL-C levels $(P=0.001)$ were significantly reduced in the $P E$ cases when compared to NP women. $50 \%$ and $47 \%$ of PE cases had intrauterine growth restriction and low birthweight infants, respectively. In conclusion, DMPA use predisposes women to a high risk of developing PE. DMPA could partly contribute to endothelial dysfunction, hyperhomocysteinaemia, dyslipidaemia and excessive weight gain, all of which characterize PE.
\end{abstract}




\section{Keywords}

Preeclampsia, Hormonal Contraceptives, Depot Medroxyprogesterone Acetate

\section{Introduction}

Hormonal contraception generally refers to birth control methods that act on the endocrine system. There are two main types of hormonal contraceptive formulations. These are combined methods that contain both estrogen and progestin, and progestogen-only methods which contain only progesterone or one of its synthetic analogues (progestins) [1]. Combined methods work by suppressing ovulation and thickening cervical mucus, while progestogen-only methods reduce the frequency of ovulation [1]. HC use is associated with hypertension, dyslipidemia, and insulin resistance, all of which characterize hypertensive disorders of pregnancy (HDP) [2].

HDP (which includes preeclampsia) is one of the commonest causes of maternal mortality [3] and accounted for 37,000 deaths in 1990 and 29,000 in 2013 [4]. Preeclampsia (PE) is characterized by hypertension and significant proteinuria [5]. The disorder occurs after 20 weeks of pregnancy and worsens over time [6]. In Ghana, the incidence of PE was reported to be 7.03\% [7]. Another study conducted in Ghana also showed that the prevalence of PE was 6.55\% [8].

Although the exact pathophysiologic mechanism is not clearly understood, PE is primarily a disorder of placental dysfunction leading to a syndrome of endothelial dysfunction with associated vasospasm. Epidemiological studies have indicated that HC may play a role in the pathogenesis of cardiovascular diseases [9]. However, the extent to which HC contributes to PE remains unknown, hence the basis of this study. Our study aimed at evaluating the use of hormonal contraceptives as a primary cause of preeclampsia (PE) among Ghanaians.

The objective of the study, therefore, was to determine the level to which DMPA could contribute to hyperhomocysteinaemia, endothelial dysfunction and PE.

\section{Methods}

\subsection{Study Design/Site}

The study was a systematic randomized case-control study conducted at Comboni Mission hospital, Sogakope, in the South Tongu District of the Volta Region of Ghana in 2019.

\subsection{Study Population}

Stat Cal utility feature of Epinfo Statistical software was used for sample size determination using confidence interval of $95 \%$ and power of $80 \%$. A minimum size of 30 cases and 30 controls was obtained. A total of 60 participants com- 
prising $30 \mathrm{PE}$ cases and 30 healthy normotensive pregnant women (control group), aged 18 - 40 years were recruited. The study participants comprised pregnant women with gestational age above 20 weeks to 40 weeks. The cases were recruited according to their history, clinical presentation and proteinuria equivalent to $1+$ or higher on a urine dipstick test. Furthermore, cases were singleton pregnant women with systolic blood pressure $\geq 140 \mathrm{~mm} \mathrm{Hg}$ and/or diastolic blood pressure $\geq 90 \mathrm{~mm} \mathrm{Hg}$.

Healthy normotensive pregnant women without any pregnancy complications or any other disease condition formed the control group. Pregnant women with the history of previous hypertension, those with twin pregnancy history and those with known vitamin deficiency (folic acid, B12) were excluded. Additionally, pregnant women suffering from diabetes, malnutrition, and those with any hepatic disorder, chronic renal disease, hypothyroidism, severe anaemia, and chronic illnesses were excluded. Cigarette smokers, alcoholics, and those on folic acid and other medication that could introduce confounding factors such as folate antagonists and nitrous oxide were also excluded.

A well-structured pre-tested questionnaire was used to obtain detailed medical, obstetric, dietary and socio-demographic data from each participant (Appendix A).

\subsection{Measurement of Blood Pressure}

In accordance with recommendations of the American Heart Association [10], the blood pressure of each participant was measured by trained personnel using a mercury sphygmomanometer (Omron M2 basic, Netherland). The procedure was repeated for each patient between 5 - 10 minutes. Mean values of duplicate measurements were recorded as the blood pressure to the nearest $2.0 \mathrm{mmHg}$.

\subsection{Anthropometric Measurements}

Weight was measured with the Omron BF-506 Body Composition Monitor (IL, USA) to the nearest $0.1 \mathrm{~kg}$ in light clothing with subjects barefooted and standing erect. Height was measured with a portable Seca stadiometer (Hamburg, Germany) to the nearest 0.1 centimeter.

\subsection{Blood Sample Collection}

About $5 \mathrm{ml}$ of venous blood sample was drawn from the antecubital vein of each participant after 8 - 14 hours overnight fast and immediately transferred into gel separator tubes. The clotted samples were centrifuged at $3000 \mathrm{rpm}$ for 5 minutes and the sera stored at $-80^{\circ} \mathrm{C}$ until assayed.

\subsection{Biochemical Analysis}

Boehringer-Mannheim dipstick proteinuria (Mannheim, Germany) was used to determine proteinuria from the urine sample of each participant. Homocysteine (HCY) and lipid profile (HDL-C, LDL-C, total cholesterol and triglycerides) 
concentrations were determined using appropriate laboratory methods.

\subsection{Determination of Serum Homocysteine}

HCY was determined by an enzymatic UV method, using Human homocysteine liquid UV diagnostics kit (Wiesbaden, Germany). The procedure was followed according to the manufacturer's instructions and the final chromogen measured at $340 \mathrm{~nm}$ on a Mindray UV spectrophotometer (Shenzhen, China).

\subsection{Determination of Serum Lipid Profile}

Total cholesterol (TC), high density lipoprotein cholesterol (HDL-C), low density lipoprotein cholesterol (LDL-C) and triglyceride (TG) were performed using BioSystem kits on an A25 BioSystem autoanalyzer (Barcelona, Spain). However, LDL was calculated as follows: $\mathrm{LDL}=\mathrm{TC}-(\mathrm{HDL}+\mathrm{TG} / 2.2) \mathrm{mmol} / \mathrm{L}$.

\subsection{Determination of Intrauterine Growth Restriction (IUGR) and Birth Weight}

Estimated foetal weight (EFW) and birthweight (BWT) were obtained from the medical record of each participant. EFW for each study participant was determined using Sonoscape S6 by a certified clinical sonologist. Using the smoothed percentiles of estimated birth weight (grams) for gestational age [11] [12], IUGR was determined using the 10th percentile.

\subsection{Statistical Analysis}

IBM SPSS Statistics for Windows, Version 21.0 (Armonk, NY: IBM Corporation) was used. Continuous variables were expressed as mean $\pm \mathrm{SD}$. The independent sample t-test and Fisher exact tests were used where appropriate to compare means of the women with PE and the control group. Pearson correlation coefficient test was used to determine the correlation between study parameters. Using a multivariate logistic regression with a $95 \%$ confidence interval $(\mathrm{CI})$, the odds ratio (OR) was determined to quantify the risk of women with $\mathrm{PIH}$ in comparison with controls. Statistical significance level was set at $\mathrm{P}<0.05$.

\section{Results}

\subsection{Socio-Demographic and Clinical Characteristics of Study Participants}

PE cases and the normal pregnant (NP) women had similar ages. The majority of the study participants were within the age range of 25 - 34 years. Other age groups' percentages were as follows: $3.3 \%, 21.7 \%, 10.0 \%$, and $3.3 \%$ for ages $>40$, $35-39,20-24$, and $<20$, respectively. About twenty-eight percent (28.3\%) of the participants had completed Junior High School, whereas 20.0\%, 20.0\% and 13.3\% had completed Senior High School, Tertiary, and Basic (Primary) education, respectively. About thirty-eight percent (38.3\%) of the pregnant women were obese, compared to $35.0 \%$ and $26.7 \%$, respectively, who were considered 
overweight and normal weight. Additionally, fifty-eight percent (58.3\%) of the study participants had normal systolic blood pressure (SBP), compared to $41.7 \%$ who had high SBP. Normal and high diastolic blood pressure (DBP) was observed in $68.3 \%$ and $31.7 \%$ of the pregnant women, respectively. The difference in the SBP and DBP of the PE cases compared to the NP women was statistically significant at $\mathrm{P}<0.05$ (Table 1).

Table 1. Socio-demographic and clinical characteristics of study participants.

\begin{tabular}{cccc}
\hline Parameters & PE $(\mathrm{n}=30)$ & NP $(\mathrm{n}=30)$ & $\begin{array}{r}\text { Total }(\mathbf{n}=60) \\
\text { PE + NP }\end{array}$ \\
\hline Age (yrs) & $30.50 \pm 5.78$ & $29.13 \pm 4.98$ & $29.82 \pm 5.3$ \\
Age category (yrs) & $1(3.3 \%)$ & $1(3.3 \%)$ & $2(3.3 \%)$ \\
$<20$ & $2(6.7 \%)$ & $4(13.3 \%)$ & $6(10.0 \%)$ \\
$20-24$ & $9(30.0 \%)$ & $10(33.3 \%)$ & $19(31.7 \%)$ \\
$25-29$ & $8(26.7 \%)$ & $10(33.3 \%)$ & $18(30.0 \%)$ \\
$30-34$ & $8(26.7 \%)$ & $5(16.7 \%)$ & $13(21.7 \%)$ \\
$35-39$ & $2(6.7 \%)$ & Nil $(0.0 \%)$ & $2(3.3 \%)$ \\
$\geq 40$ & & &
\end{tabular}

\section{Educational Level}

$\begin{array}{lccc}\text { No Education } & 8(26.7 \%) & 3(10.0 \%) & 11(18.3 \%) \\ \text { Basic } & 6(20.0 \%) & 2(6.7 \%) & 8(13.3 \%) \\ \text { JHS } & 8(26.7 \% & 9(30.0 \%) & 17(28.3 \%) \\ \text { SHS } & 5(16.7 \%) & 7(23.3 \%) & 12(20.0 \%) \\ \text { Tertiary } & 3(10.0 \%) & 9(30.0 \%) & 12(20.0 \%)\end{array}$

BMI

$\begin{array}{lccc}\text { Normal } & 7(23.3 \%) & 9(30.0 \%) & 16(26.7 \%) \\ \text { Overweight } & 8(26.7 \%) & 13(43.3 \%) & 21(35.0 \%) \\ \text { Obese } & 15(50.0 \%) & 8(26.7 \%) & 23(38.3 \%)\end{array}$

SBP

$\begin{array}{llll}\text { Normal } & 5(16.7 \%)^{*} & 30(100.0 \%) & 35(58.3 \%) \\ \text { High } & 25(83.3 \%) & \text { Nil }(0.0 \%) & 25(41.7 \%)\end{array}$

DBP

$\begin{array}{lccc}\text { Normal } & 11(36.7 \%)^{*} & 30(100.0 \%) & 41(68.3 \%) \\ \text { High } & 19(63.3 \%) & \text { Nil }(0.0 \%) & 19(31.7 \%)\end{array}$

Chi-square or Fischer exact test whenever $\mathrm{n}<5$ was used to compare the PE group with the NP group. ${ }^{*} \mathrm{p}<$ 0.05 was considered a statistically significant difference. BMI of $19 \mathrm{Kg} / \mathrm{m}^{2}-24.9 \mathrm{Kg} / \mathrm{m}^{2}, 25.0 \mathrm{Kg} / \mathrm{m}^{2}-29.9$ $\mathrm{Kg} / \mathrm{m}^{2}$ and $>30 \mathrm{Kg} / \mathrm{m}^{2}$ were categorized as normal, overweight and obese respectively; PE: Preeclampsia; NP: Normal pregnant control; JHS: Junior high school; SHS: Senior high school; BMI: Body Mass Index; SBP: Systolic Blood Pressure; DBP: Diastolic Blood Pressure. 


\subsection{The Clinical Variables of the Study Population}

The clinical variables of the study population are shown in Table 2. There was no statistically significant difference in the mean age between the PE cases and NP women. Furthermore, BMI, SBP, DBP, EFW, BWT, TG, LDL-C and HDL-C of the $\mathrm{PE}$ cases were significantly different $(\mathrm{P}<0.05)$ from the NP women. Gestational age and total cholesterol concentrations however, showed no statistically significant differences. Majority (65.0\%) of the pregnant women were within 34 - 37 weeks of gestation.

The highest percentage (28.3\%) of participants were primiparous, whereas $26.7 \%, 21.7 \%, 15.0 \%$ and $8.3 \%$ were nulliparous, secundiparous, tertiparous and multiparous respectively. Majority (30.0\%) of the participants were multigravida

About fifty-seven percent (56.7\%) of the pregnant women were never on any contraceptive prior to the pregnancy; whereas $43.3 \%$ once used DMPA. Of the PE cases, $80.0 \%$ used DMPA, prior to getting pregnant. About twelve percent (11.7\%) of the study participants had a family history of hypertension, with the majority amongst the PE cases (Table 3). There was IUGR if the estimated foetal weight (EFW) was below the 10th percentile for their gestational age. None of the normal pregnant (NP) women had IUGR, while $50.0 \%$ of the PE patients

Table 2. The clinical variables of the study population.

\begin{tabular}{cccc}
\hline Parameters & PE $(\mathrm{n}=30)$ & NP $(\mathrm{n}=30)$ & $P$-value \\
\hline Age (years) & $30.50 \pm 5.78$ & $29.13 \pm 4.98$ & 0.330 \\
Gestational Age (yrs) & $34.50 \pm 2.83$ & $34.63 \pm 2.00$ & 0.834 \\
Weight (Kg) & $84.07 \pm 21.33$ & $72.20 \pm 12.69$ & 0.011 \\
Height m & $1.62 \pm 0.09$ & $1.56 \pm 0.12$ & 0.029 \\
BMI (Kg/m $)$ & $30.35 \pm 5.71$ & $27.93 \pm 4.02$ & 0.063 \\
SBP (mmHg) & $155.67 \pm 15.30$ & $112.33 \pm 10.06$ & $<0.0001$ \\
DBP (mmHg) & $99.33 \pm 10.81$ & $73.67 \pm 9.64$ & $<0.0001$ \\
EFW (Kg) & $2.00 \pm 0.62$ & $3.00 \pm 0.40$ & $<0.0001$ \\
BWT (Kg) & $2.50 \pm 0.36$ & $3.46 \pm 0.62$ & $<0.0001$ \\
TC (mmol/L) & $4.17 \pm 1.48$ & $3.64 \pm 1.45$ & 0.162 \\
TRG (mmol/L) & $1.24 \pm 0.84$ & $0.85 \pm 0.33$ & 0.024 \\
LDL (mmol/L) & $2.92 \pm 1.54$ & $2.15 \pm 1.03$ & 0.026 \\
HDL (mmol/L) & $0.87 \pm 0.50$ & $1.31 \pm 0.46$ & 0.001 \\
Homocysteine ( $\mu$ mol/L) & $32.35 \pm 11.08$ & $9.32 \pm 2.73$ & $<0.0001$ \\
\hline
\end{tabular}

TC: serum cholesterol; TRG: triglycerides; LDL: Low Density Lipoprotein cholesterol; HDL: High Density Lipoprotein cholesterol. Data was expressed as mean \pm standard deviation (SD). Independent (unpaired) $\mathrm{t}$-test was to compare means of the clinical variables between the preeclamptic patients and the normal pregnant women. 
Table 3. Obstetric history of studied participants.

\begin{tabular}{lcc}
\hline Parameters & PE $(\mathrm{n}=30)$ & Total $(\mathrm{n}=60)$ \\
$\mathrm{PE}+\mathrm{NP}$
\end{tabular}

OBSTETRIC HISTORY

\section{Gestational Age at sampling}

$\begin{array}{lccc}<34 \text { weeks } & 9(30.0 \%) & 8(26.7 \%) & 17(28.3 \%) \\ 34 \text { - } 37 \text { weeks } & 18(60.0 \%) & 21(70.0 \%) & 39(65.0 \%) \\ \geq 38 \text { weeks } & 3(10.0 \%) & 1(3.3 \%) & 4(6.7 \%)\end{array}$

Parity

Nulliparous

Primipara

Secundipara

Tertipara

Multipara $(\geq 4)$

\section{Gravidity}

Primigravida
Secungravida
Tertigravida
Multigravida ( $\geq 4)$

Type of contraceptive used

DMPA

No contraceptive used

Family history of HTN

Yes

Previous obstetric history

No complication
Induced abortion
Spontaneous abortion
Miscarriage

Foetal growth restriction

$\begin{array}{llll}\text { Yes } & 15(50.0 \%) & \text { Nil }(0.0 \%) & 15(25.0 \%) \\ \text { No } & 15(50.0 \%) & 30(100.0 \%) & 45(75.0 \%)\end{array}$

\section{Birth weight}

$\begin{array}{llll}\text { Normal weight } & 16(53.3 \%) & 30(100.0 \%) & 46(76.7 \%) \\ \text { Underweight } & 14(46.7 \%) & \text { Nil }(0.0 \%) & 14(23.3 \%)\end{array}$

DMPA: Depot medroxyprogesterone acetate; HTN: Hypertension. 
demonstrated IUGR. Babies who weighed $<2.5 \mathrm{Kg}$ at birth were considered underweight babies while those weighing $>2.5 \mathrm{Kg}$ at birth were considered normal weight babies. While none of the NP women gave birth to underweight babies, $46.7 \%$ of the babies by PE women were underweight (Table 3). Positive correlations for PE were as follows: SBP and DBP $(\mathrm{p}=0.05 ; \mathrm{r}=0.858)$; GA and BWT ( $=0.05, \mathrm{r}=0.425)$; TG and SBP $(\mathrm{p}=0.05, \mathrm{r}=0.457)$; TG and TC $(\mathrm{p}=0.05, \mathrm{r}=$ 0.397); TG and LDL ( $\mathrm{p}=0.05, \mathrm{r}=0.397)$; LDL and TC ( $\mathrm{p}=0.001, \mathrm{r}=0.908)$; HCY and DBP $(p=0.05, r=0.441)$. Negative correlations for PE were as follows: GA and EFW ( $\mathrm{p}=0.05, \mathrm{r}=-0.403)$; GA and BWT ( $\mathrm{p}=0.05, \mathrm{r}=-0.337)$; BWT and DBP $(\mathrm{p}=0.05, \mathrm{r}=-0.366)$; B12 and HDL ( $\mathrm{p}=0.05, \mathrm{r}=-0.368)$; Folate and $\mathrm{TC}(\mathrm{p}=0.05 ; \mathrm{r}=-0.414)$.

\subsection{Multivariate Logistic Regression of Factors Associated with PE}

The use of HC prior to pregnancy was associated with a 30-fold (29.71) increase in the odds of developing PE (Table 4).

\section{Discussion}

\subsection{Main Findings}

In this study, the risk of developing PE among women who used DMPA, prior to pregnancy was assessed. The findings of this study showed that $80.0 \%$ of the preeclamptic patients have at some point in their life-time used DMPA. The use of DMPA prior to pregnancy was associated with about thirty-fold increase in the odds of developing PE $(\mathrm{OR}=29.71, \mathrm{p}<0.001)$. The study demonstrated that serum homocysteine and lipid profile levels were significantly altered with the use of HC.

\subsection{Interpretation of Results}

Depot medroxyprogesterone acetate (DMPA), an injectable progestin-only contraceptive, is a popular contraceptive of choice, particularly for younger premenopausal women because of the ease of use and high compliance. Ultimately, DMPA is a potent contraceptive with consequences [13] [14] [15].

Table 4. Multivariate logistic regression of factors associated with PE.

\begin{tabular}{cccc}
\hline Variable & OR & $(95 \% \mathrm{CI})$ & P-value \\
\hline Contraceptive use & & & \\
Yes & 26.00 & $8.12-83.25$ & $<0.001$ \\
No $^{*}$ & 1.00 & - & - \\
Type of contraceptive used & & - & - \\
None & 1.00 & $9.01-97.96$ & $<0.001$ \\
DMPA & 29.71 &
\end{tabular}

OR: Odds Ratio; CI: Confidence Interval; Reference group. 
Estrogens are known to have a cardio-protective influence in women by improving endothelial function and low-density lipoprotein (LDL) concentrations while decreasing concentrations of endothelin-1 (ET-1) and homocysteine. However, DMPA has been shown to counteract the beneficial effects of estrogens in animals and in some studies, in postmenopausal women [13]. DMPA creates a hypoestrogenic effect, hence diminishing estrogen's protective role on blood vessels [14].

Furthermore, it has been indicated that using HC pills can adversely affect homocysteine [16]. As a result, the significant hyperhomocysteinaemia and dyslipidaemia observed in the preeclamptic women might partly be due to the hypoestrogenic effect of DMPA.

Endothelial dysfunction plays a critical role in the pathogenesis of PE [17]. Widespread endothelial dysfunction is hypothesized to mediate the characteristic generalized vasospasm manifesting as hypertension, a central feature of established PE [18]. In a study where the authors evaluated DMPA damage to blood vessels via flow-mediated dilation [(FMD)-FMD measures endothelial function], the data showed significant differences in FMD among DMPA users and non-hormonal contraceptive users as well as combined oral contraceptive (COC) users and non-hormonal contraceptive users [19]. Between the two HC groups, there was no significant difference in FMD. Data of the study group showed that DMPA users also had lower total cholesterol as well as lower LDL-C, whereas non-hormonal contraceptive users had a higher HDL-C. The findings of Meendering and his colleagues [13] support previous literature that suggests that oral progesterone (which may be considered to have properties similar to DMPA) has androgenic effects that are harmful to the cardiovascular system.

Although the antioxidant status and markers of oxidative stress were not assessed in this study, DMPA is known to induce ovarian oxidative stress [20]. A study to determine the protective effect of vitamins $C$ and $E$ on DMPA-induced ovarian oxidative stress in vivo, found elevated levels of malondialdehyde and reduced level of superoxide dismutase in DMPA users when compared to the control group [20]. The findings of that study indicate that DMPA increases lipid peroxidation of ovarian tissue. As a result, oxidative stress resulting from the use of DMPA may play a pivotal role in endothelial cell dysfunction in preeclampsia [21].

Hyperhomocysteinaemia is recognized as an important and independent risk factor for cardiovascular diseases [22]. HC increases homocysteine concentration, leading to hyperhomocysteinaemia [16]. Consequently, the presence of significant hyperhomocysteinaemia in the preeclamptic women when compared to normal healthy pregnant women in this study may partly be due to the use of DMPA by majority of the women with preeclampsia prior to pregnancy. This may further have contributed to the development of PE.

Other cardiovascular risk markers were significantly altered in women with 
PE than in the healthy normotensive pregnant women in this study. The preeclamptic women demonstrated significant dyslipidemia in comparison with the normal pregnant women. Although studies that assessed lipid profile in pregnant women who used progestin-only contraceptive prior to pregnancy remain scarce, data on women who used Combined Oral Contraceptive (COC) indicated that $\mathrm{COC}$ uptake results in a more atherogenic lipid profile by increasing LDL-C and decreasing HDL-C concentrations [23]. It has also been suggested that perhaps free radicals produced during metabolism of hormonal contraceptives change the amounts of atherogenic lipids [24].

Furthermore, weight gain is a commonly perceived side effect of hormonal contraception [24]. Our study showed that the preeclamptic women had a higher BMI than the control group (NP), though statistically insignificant. Given the serious public health problem posed by overweight and obesity worldwide [15], the public health impact of any contraceptive on obesity could be substantial. Weight gain and changes in body composition have previously been reported among DMPA users [25]. In a 36-months longitudinal study of 703 women taking contraceptives, it was observed that DMPA users had a $5.1 \mathrm{Kg}$ weight gain when compared to the $1.5 \mathrm{Kg}$ weight gain among oral contraceptive users and the $2.1 \mathrm{Kg}$ weight gain among non-hormonal contraceptives users [26]. A meta-analysis of maternal BMI and PE showed that the risk of developing PE was doubled with every 5 to 7 unit increase in pre-pregnancy BMI [27]. Moreover, some authors reported that the risk of preeclampsia during pregnancy doubled in overweight women while it was 4.5 times higher in obese women [27]. Consequently, changes in weight resulting from the use of DMPA further increases the risk of developing PE in later life.

\subsection{Strengths and Limitations of Study}

The small sample size used is a limitation of this study. Nevertheless, this research adds a lot to the existing knowledge, as there is paucity of data on the role DMPA in the aetiology of PE.

\section{Conclusion}

Overall, the study demonstrated that the use of DMPA prior to pregnancy is a primary cause of PE. DMPA could partly contribute to endothelial dysfunction, hyperhomocysteinaemia, dyslipidaemia and excessive weight gain, all of which contribute to the development of PE. Women who decide to use DMPA should be well educated on the benefits and harmful effects of this contraceptive.

\section{Acknowledgements}

The researchers are grateful to the entire staff and management of Comboni Hospital, most especially, Dr. Momudu Cham, Mr. Stephen Adusu and Miss Veronica Perko for the partnership and permission granted to conduct this study. 


\section{Contribution to Authorship}

All authors collaborated to carry out this work. Authors L. Asare, W. K. B. A. Owiredu, C. Obikorang and G. A. Asare conceptualized and designed the study. Participants recruitment and data generation were carried out by authors L. Asare, W. Tashie, L. Seidu and G. A. Asare. Authors L. Asare, W. K. B. A. Owiredu, C. Obikorang, W. Tashie and G. A. Asare analyzed the data. Authors L. Asare, W. K. B. A. Owiredu, C. Obikorang, W. Tashie, L. Seidu, G. A. Asare drafted the manuscript. Authors L. Asare, W. K. B. A. Owiredu, C. Obikorang, E. Appiah and G. A. Asare reviewed the manuscript for intellectual content and each author approved the final manuscript.

\section{Ethical Approval}

Ethical approval for the study was granted by the facility authorized committee in a letter (KAD/CHS/ADM/026/2014]).

\section{Consent}

Informed consent was obtained from participants before enrollment into this study.

\section{Conflicts of Interest}

The authors declare no conflicts of interest regarding the publication of this paper.

\section{References}

[1] Rivera, R., Yacobson, I. and Grimes, D. (1999) The Mechanism of Action of Hormonal Contraceptives and Intrauterine Contraceptive Devices. American Journal of Obstetrics \& Gynecology, 181, 1263-1269. https://doi.org/10.1016/S0002-9378(99)70120-1

[2] Thadhani, R., Stampfer, M.J., Hunter, D.J., Manson, J.E., Solomon, C.G. and Curhan, G.C. (1999) High Body Mass Index and Hypercholesterolemia: Risk of Hypertensive Disorders of Pregnancy. Obstetrics \& Gynecology, 94, 543-550. https://doi.org/10.1097/00006250-199910000-00011

[3] Arulkumaran, N. and Lightstone, L. (2013) Severe Pre-Eclampsia and Hypertensive Crises. Best Practice \& Research: Clinical Obstetrics \& Gynaecology, 27, 877-884. https://doi.org/10.1016/j.bpobgyn.2013.07.003

[4] Global Burden of Disease Study 2013 Collaborators (2015) Global, Regional, and National Incidence, Prevalence, and Years Lived with Disability for 301 Acute and Chronic Diseases and Injuries in 188 Countries, 1990-2013: A Systematic Analysis for the Global Burden of Disease Study 2013. The Lancet (London, England), 386, 743-800.

[5] Eiland, E., Nzerue, C. and Faulkner, M. (2012) Preeclampsia. Journal of Pregnancy, 2012, Article ID: 586578. https://doi.org/10.1155/2012/586578

[6] Al-Jameil, N., Khan, F.A., Khan, M.F. and Tabassuma, H.A. (2014) Brief Overview of Preeclampsia. Journal of Clinical Medicine Research, 6, 1-7. https://doi.org/10.4021/jocmr1682w 
[7] Obed, S. and Aniteye, P. (2006) Birth Weight and Ponderal Index in Pre-Eclampsia: A Comparative Study. Ghana Medical Journal, 40, 8-13.

[8] Ahenkorah, L. (2009) Metabolic Syndrome, Oxidative Stress and Putative Risk Factors amongst Ghanaian Women Presenting with Pregnancy-Induced Hypertension. Department of Molecular Medicine, School of Medical Sciences, Kwame Nkrumah University of Science \& Technology, Kumasi.

[9] Asare, G.A., Santa, S., Ngala, R., Asiedu, B., Afriyie, D. and Amoah, A.G.B. (2014) Effect of Hormonal Contraceptives on Lipid Profile and the Risk Indices for Cardiovascular Disease in a Ghanaian Community. International Journal of Women's Health, 6, 597-603. https://doi.org/10.2147/IJWH.S59852

[10] Kirkendall, W.M., Burton, A.C., Epstein, F.H. and Freis, E.D. (1967) Recommendations for Human Blood Pressure Determination by Sphygmomanometers. Circulation, 36, 980-988. https://doi.org/10.1161/01.CIR.36.6.980

[11] Arias, F., Daftary, N.S. and Bhide, G.A. (2008) Practical Guide to High-Risk Pregnancy and Delivery. Elsevier, New Delhi.

[12] Leveno, J.K. (2013) Williams Manual of Pregnancy Complications. 23rd Edition, The McGraw-Hill Companies, Inc., New York.

[13] Meendering, J.R., Torgrimson, B.N., Miller, N.P., Kaplan, P.F. and Minson, C.T. (2008) Estrogen, Medroxyprogesterone Acetate, Endothelial Function, and Biomarkers of Cardiovascular Risk in Young Women. The American Journal of Physiology-Heart and Circulatory Physiology, 294, H1630-H1637.

https://doi.org/10.1152/ajpheart.01314.2007

[14] Dudar, A.K. and Khadijih, A. (2010) Evaluating Use of Depo-Provera: A Closer Look at Association with Skeletal, Cardiovascular and Metabolic Systems. The University of Toledo Digital Repository, The University of Toledo, Toledo.

[15] Batista, G.A., Souza, A.L., Marin, D.M., Sider, M., Melhado, V.C., Fernandes, A.M. and Alegre, S.M. (2016) Body Composition, Resting Energy Expenditure and Inflammatory Markers: Impact in Users of Depot Medroxyprogesterone Acetate after 12 Months Follow-Up. Archives of Endocrinology and Metabolism, 61, 70-75. https://doi.org/10.1590/2359-3997000000202

[16] Fallah, S., Nouroozi, V., Seifi, M., Samadikuchaksaraei, A. and Mokhtarned-Aghdashi, E. (2012) Influence of Oral Contraceptive Pills on Homocysteine and Nitric Oxide Levels: As Risk Factors for Cardiovascular Disease. Journal of Clinical Laboratory Analysis, 26, 120-123. https://doi.org/10.1002/jcla.21492

[17] Adu-Bonsaffoh, K., Antwi, D.A., Obed, S.A. and Gyan, B. (2015) Nitric Oxide Dysregulation in the Pathogenesis of Preeclampsia among Ghanaian Women. Integrated Blood Pressure Control, 8, 1-6. https://doi.org/10.2147/IBPC.S68454

[18] Braekke, K., Ueland, P.M., Harsem, N.K. and Staff, A.C. (2009) Asymmetric Dimethylarginine in the Maternal and Fetal Circulation in Preeclampsia. Pediatric Research, 66, 411-415. https://doi.org/10.1203/PDR.0b013e3181b33392

[19] Lizarelli, P.M., Martins, W.P., Vieira, C.S., Soares, G.M., Franceschini, S.A. and Ferriani, R.A. (2009) Both a Combined Oral Contraceptive and Depot Medroxyprogesterone Acetate Impair Endothelial Function in Young Women. Contraception, 79, 35-40. https://doi.org/10.1016/j.contraception.2008.07.024

[20] Ismiyati, A., Wiyasa, I.W. and Hidayati, D.Y. (2016) Protective Effect of Vitamins C and E on Depot-Medroxyprogesterone Acetate-Induced Ovarian Oxidative Stress in Vivo. Journal of Toxicology, 2016, Article ID: 3134105. https://doi.org/10.1155/2016/3134105

[21] Gupta, S., Agarwal, A. and Sharma, R.K. (2005) The Role of Placental Oxidative 
Stress and Lipid Peroxidation in Preeclampsia. Obstetrical \& Gynecological Survey, 60, 807-816. https://doi.org/10.1097/01.ogx.0000193879.79268.59

[22] Mahal, M., Yeasmin, F., Amin, S., Shahnaj, A., Rashid, M. and Hossain, M.S. (2009) Association of Serum Homocysteine and Serum Lipid with Eclampsia. JAFMC Bangladesh, 5, 7-10. https://doi.org/10.3329/jafmc.v5i1.2842

[23] Fallah, S., Sanjary, P.M., et al. (2012) Adiponectin, Leptin and Lipid Profiles Evaluation in Oral Contraceptive Pill Consumers. Archives of Gynecology and Obstetrics, 285, 1747-1752. https://doi.org/10.1007/s00404-011-2192-3

[24] Fallah, S. and Sanjary, M. (2015) The Effects of Hormonal Contraceptives on the Various Biomarkers Levels: A Review. IJBRITISH, 2, 131-171.

[25] Mishell, D.R. (1996) Pharmacokinetics of Depot Medroxyprogesterone Acetate Contraception. The Journal of Reproductive Medicine, 41, 381-390.

[26] Ashley, M., Nault, B.S., Jeffrey, F., Peipert, Z.Q. and Madden, T. (2013) Validity of Perceived Weight Gain in Women Using Long-Acting Reversible Contraception and Depot Medroxyprogesterone Acetate. American Journal of Obstetrics \& Gynecology, 208, 48. https://doi.org/10.1016/j.ajog.2012.10.876

[27] Vinod, T., Latti, R.G., et al. (2015) Correlation between Maternal Body Mass Index and Incidence of Pregnancy Induced Hypertension. Indian Journal of Basic and Applied Medical Research, 4, 86-90. 


\section{Appendix A}

\section{Research Questionnaire}

Personal History

Identification number:

Name:

Residential area:

Health Facility:

Marital Status:

Obstetric History

Gestational Age (Weeks).

Parity:

Weight:

(Kgs)

Body Mass Index:

Have you been pregnant before?

If yes, number of pregnancies.

Still births

Induced abortion

Preterm delivery

Live births

Birth weight

Intrauterine Growth Restriction

\section{Medical History}

Do you have any of these?

Pre-eclampsia

Heart disease

Hypertension

Kidney disease

Liver diseases

Diabetes mellitus

Sickle cell disease

Severe anaemia

Folic acid deficiency

Vitamin $B_{12}$ deficiency

Have you ever being diagnosed of any of the above condition before this pregnancy?

Yes

Yes

Yes

Yes

Yes $\square$

Yes

Yes $\square$

Yes $\square$

Yes $\square$

Yes $\square$

Yes years.

No:

Mobile No:

ducational background:

Expected Date of Delivery:

ravidity:

lood pressure:

Yes

No

$(\mathrm{mmHg})$

Miscarriages:

Abortion:

Multiple births

If yes which?

When was the first time you were diagnosed of this condition?

Are you currently on any medications or injections? Yes $\square$

If yes, what are they?

Any other health complication?

Do you have any history of alcoholism? Yes

No $\square$

Have you currently drunk alcohol?

Yes

No

If yes, how long did you drink?

Have you ever smoked?

Yes

No 
If yes, how long did you smoke?

Have you ever used any contraceptive prior to this pregnancy?

Yes $\square$

No

If yes which?

Family History

Do you have family history of any of the following conditions?

Pre-eclampsia

Heart disease

Yes $\square$

No $\square$

Yes

No $\square$

Hypertension

Yes $\square$

No $\square$

Kidney disease

Yes $\square$

No $\square$

Liver diseases

Yes $\square$

No $\square$

Diabetes mellitus

Yes $\square$

No $\square$

Sickle cell disease

Yes $\square$

No $\square$

Multiple pregnancies

Yes $\square$

No $\square$

Birth defects (specify)

Yes

No $\square$

\section{Nutritional Data}

\begin{tabular}{ccccc}
\hline & $\begin{array}{c}\text { How often do you eat } \\
\text { the following foods }\end{array}$ & None Daily & Twice a week & Weekly \\
\hline I & Meat \\
II & Fish \\
III $\quad$ Green leafy vegetables & \\
\hline
\end{tabular}

